\title{
Autonomic neuropathy as post-acute sequela of SARS-CoV-2 infection: a case report
}

\author{
Shruti P. Agnihotri ${ }^{1} \cdot$ Carmela V. San Luis $^{1} \cdot$ Mohamed Kazamel $^{1}$ (i)
}

Received: 29 October 2021 / Revised: 13 January 2022 / Accepted: 27 January 2022 / Published online: 18 February 2022

(c) Journal of NeuroVirology, Inc. 2022

\begin{abstract}
Symptoms of autonomic dysfunction, particularly those of orthostatic intolerance, continue to represent a major component of the currently recognized post-acute sequelae of SARS-CoV-2 infections. Different pathophysiologic mechanisms can be involved in the development of orthostatic intolerance including hypovolemia due to gastrointestinal dysfunction, fatigueassociated deconditioning, and hyperadrenergic state due to pandemic-related anxiety. Additionally, there has been a wellestablished association of a common primary autonomic disorder like postural orthostatic tachycardia syndrome, a subtype of orthostatic intolerance, with antecedent viral infections. Here we report a case of neuropathic type postural orthostatic tachycardia syndrome as a form of autonomic neuropathy that developed following COVID-19 infection.
\end{abstract}

Keywords Autonomic neuropathy · COVID-19 - Orthostatic intolerance · Postural orthostatic tachycardia syndrome · Small fiber neuropathy $\cdot$ Valsalva maneuver

\section{Introduction}

The term "orthostatic intolerance" (OI) describes the development of symptoms of cerebral hypo-perfusion and/or sympathetic overactivity when assuming an upright position that subsequently improves on recumbence. OI encompasses more defined conditions like orthostatic hypotension $(\mathrm{OH})$, syncope with or without $\mathrm{OH}$, and postural orthostatic tachycardia syndrome (POTS) (Gould et al. 2021). POTS is defined as a clinical syndrome that features chronic symptoms of OI with excessive sinus tachycardia upon standing, in the absence of $\mathrm{OH}$ (Freeman et al. 2011). Symptoms of OI after COVID-19 infections are becoming widely recognized as the COVID-19 pandemic continues. Recently, the American Autonomic Society identified important research questions on the topic including whether these patients have objective evidence of abnormalities on formal autonomic testing or any other pathophysiological abnormalities (Raj et al. 2021). We herein describe a case of neuropathic type

Mohamed Kazamel

mkazamel@uabmc.edu

1 Department of Neurology, University of Alabama at Birmingham, 1720 2nd Ave. South, SC271, Birmingham, AL 35294-0017, USA
POTS with detailed autonomic testing data, that developed following COVID-19 infection.

\section{Case report}

A 47-year-old woman developed nasal congestion, fever, ageusia, and anosmia in whom SARS-CoV-2 was detected by RT-PCR from a nasopharyngeal swab. She did not require hospitalization. Within days, she noticed lightheadedness, palpitations, persistent hand and feet tingling and burning pain, hyperhidrosis, tremulousness, and dusky red discoloration of the feet on standing. She had no syncopal events. She was prescribed a small dose of metoprolol that helped with her palpitations. She did not report taking any new medication prior to the onset of symptoms. The patient's COMPASS-31 score was 58 when she presented to our clinic 8 months from the onset of symptoms (Sletten et al. 2012). The neurological exam was normal except for hyperalgesia below bilateral mid-shins.

Laboratory work-up for acquired causes of neuropathy including differential blood counts, comprehensive metabolic panel, A1c, thyroid function tests, vitamin B1 level, folate level, vitamin B12 level, serum protein electrophoresis, serum and urine immunofixation, celiac disease serology, paraneoplastic antibody panel, and rheumatologic markers 
was normal. HIV titers were negative. Twelve-lead EKG was normal. Bilateral lower limb nerve conduction and electromyography studies were normal. Metoprolol was stopped 2 days before autonomic testing (Fig. 1), which showed absent late phase II of the blood pressure (BP) response to Valsalva maneuver (VM) indicating sympathetic vasomotor impairment. The heart rate (HR) responses to deep breathing and VM were normal. Quantitative sudomotor axonal reflex testing (QSART) showed reduced sweat output in the forearm $\left(0.08 \mu \mathrm{L} / \mathrm{cm}^{2}\right.$, normal $\left.=0.13 \mu \mathrm{L} / \mathrm{cm}^{2}\right)$ and foot $\left(0.04 \mu \mathrm{L} / \mathrm{cm}^{2}\right.$, normal $=0.14 \mu \mathrm{L} / \mathrm{cm}^{2}$ ) sites (Sletten et al. 2015), reflecting a postganglionic sympathetic cholinergic sudomotor impairment due to autonomic neuropathy. There was no significant drop in her BP during a 10-min head-up tilt table (HUT) test. HR showed a persistent tachycardiac response in the second half of the tilt period (from $81 \mathrm{bpm}$ at baseline to an average of $117 \mathrm{bpm}$ during the last $4 \mathrm{~min}$ of the tilt period) coinciding with OI symptoms including palpitations, worsening headache, and lightheadedness. We diagnosed her with neuropathic type POTS based on the reduced QSART response and sympathetic vasomotor impairment (Jacob et al. 2000). Skin punch biopsy showed normal epidermal nerve fiber density (ENFD) for age and sex at the ankle $(12.2 / \mathrm{mm}$, normal $=5.7 / \mathrm{mm}$ (Lauria et al. 2010)) and thigh $(12.6 / \mathrm{mm})$ sites. There was a single small perivascular mononuclear cellular infiltrate surrounding a dermal small blood vessel at the thigh site which is of uncertain significance (Fig. 2). We educated the patient on the non-pharmacologic management of POTS (Bryarly et al. 2019), continued the metoprolol, and added a small dose of midodrine. Her COMPASS-31 score went down to 48 on a 3-month follow-up with most of the improvement seen in the OI and vasomotor domains.
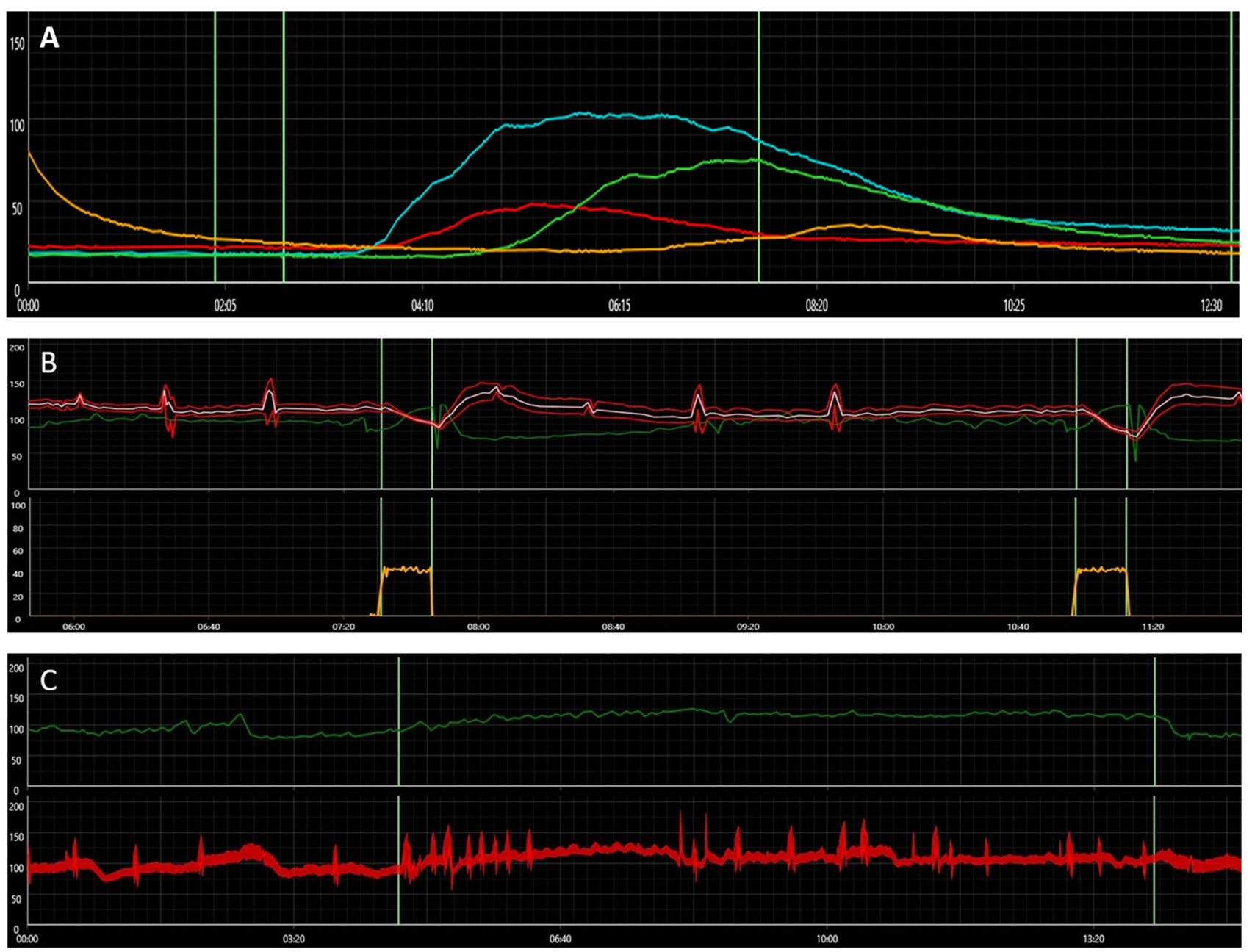

Fig. 1 A quantitative sudomotor axonal reflex test showing reduced sweat outputs in the forearm (red) and foot (yellow) sites, while it is normal in the proximal and distal leg sites (blue and green curves, respectively). B Blood pressure response to Valsalva maneuver showing diminished late phase II with normal heart rate response. C Orthostatic tachycardiac response to head-up tilt table test (upper panel) with no significant change in blood pressure (lower panel) 

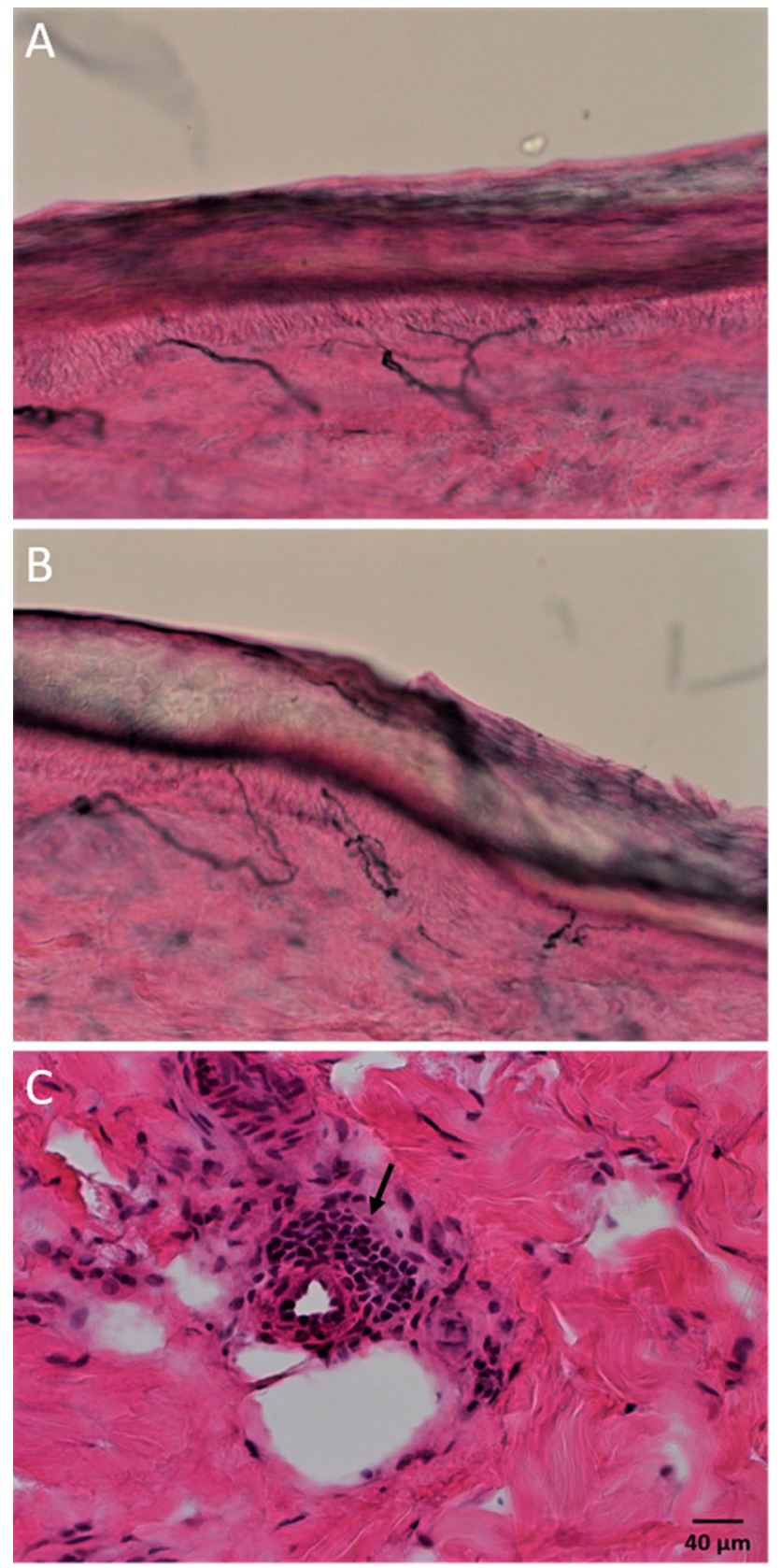

Fig. 2 Skin punch biopsy sections immunohistochemically stained with PGP 9.5 showing normal epidermal nerve fiber densities at the ankle (A) and thigh (B) sites. C Hematoxylin and eosin-stained section from the thigh site showing a small mononuclear cellular infiltrate surrounding a dermal small blood vessel (arrow)

\section{Discussion}

There are several reasons why autonomic dysfunction is expected with or following COVID-19. It had been reported in $50 \%$ of the severe acute respiratory syndrome (SARS) patients studied during the 2002 SARS epidemic (Lo et al. 2005). There is a significant association of a common primary autonomic disorder like POTS with antecedent or concomitant infections (Schondorf 1993). Furthermore, other superimposed factors could contribute to the development or worsening of orthostatic tachycardia. Patients infected with SARS-CoV-2 can develop a wide range of clinical manifestations including fatigue and gastrointestinal dysfunction. These in turn typically result in deconditioning and hypovolemia, respectively, which are known to predispose to OI. The pandemic-related anxiety and preventive measures such as quarantining could predispose the patient to deconditioning and/or hyperadrenergic state that may induce or worsen orthostatic tachycardia (Wang et al. 2021). Our patient started to have symptoms right after the infection and had sudomotor and adrenergic impairment which all make deconditioning a less likely pathophysiologic mechanism in this case.

A large retrospective study of hospitalized COVID-19 patients showed that the prevalence of autonomic dysfunction was $2.5 \%$ (Romero-Sanchez et al. 2020). A more focused retrospective study that included 27 patients who had COVID-19 infection and formal autonomic testing (median time between the onset of COVID-19 symptoms and autonomic testing was 119 days) showed abnormalities in sudomotor testing in 36\%, cardiovagal function in $27 \%$, and cardiovascular adrenergic function in $7 \%$ of patients. The most common diagnosis was subjective OI (defined as OI without demonstrable orthostatic tachycardia or $\mathrm{OH}$ ), established in $41 \%$ of patients. Orthostatic symptoms with borderline HUT findings (borderline HR increase or BP drop) were observed in $11 \%$. Only $22 \%$ of patients fulfilled the diagnostic criteria for POTS, and one patient was assigned to the neuropathic POTS category based on $4 \%$ distal anhidrosis on thermoregulatory sweat test with intact QSART and sympathetic cardiovascular testing. Another patient showed significant sudomotor abnormalities and mild cardiovascular adrenergic impairment with no symptoms or significant tachycardiac response on HUT testing (Shouman et al. 2021). Another case series of six COVID-19 patients with autonomic dysfunction reported $\mathrm{OH}$ and abnormal $\mathrm{BP}$ response to $\mathrm{VM}$ in one patient. Another patient showed reduced sweat volume in the foot site on QSART with normal volumes elsewhere in addition to a 60-bpm increase in HR during HUT. Evidence of both sympathetic adrenergic and cholinergic dysfunction did not coexist in any patient (Goodman et al. 2021).

Apart from the commonly reported post-COVID-19 OI and POTS of the hypovolemic, deconditioning, or hyperadrenergic types, this case features a neuropathic type POTS as a form of autonomic neuropathy. This is based on the observed sympathetic adrenergic and cholinergic impairments, and orthostatic tachycardia that have continued for 8 months. The reduced QSART response reflects an impairment in the small nerve fiber autonomic function. This case underscores the need for increased awareness of the varied long-lasting autonomic manifestations seen after COVID-19 infections. 


\section{Declarations}

Conflict of interest The authors declare no competing interests.

\section{References}

Bryarly M, Phillips LT, Fu Q, Vernino S, Levine BD (2019) Postural orthostatic tachycardia syndrome: JACC Focus Seminar. J Am Coll Cardiol 73(10):1207-1228. https://doi.org/10.1016/j.jacc. 2018.11.059

Freeman R, Wieling W, Axelrod FB, Benditt DG, Benarroch E, Biaggioni I, Cheshire WP, Chelimsky T, Cortelli P, Gibbons CH, Goldstein DS, Hainsworth R, Hilz MJ, Jacob G, Kaufmann H, Jordan J, Lipsitz LA, Levine BD, Low PA, Mathias C, Raj SR, Robertson D, Sandroni P, Schatz I, Schondorff R, Stewart JM, van Dijk JG (2011) Consensus statement on the definition of orthostatic hypotension, neurally mediated syncope and the postural tachycardia syndrome. Clin Auton Res 21(2):69-72. https://doi.org/10.1007/s10286-011-0119-5

Goodman BP, Khoury JA, Blair JE, Grill MF (2021) COVID-19 dysautonomia. Front Neurol 12:624968. https://doi.org/10.3389/fneur. 2021.624968

Gould SJ, Cochrane GD, Johnson J, Hebson CL, Kazamel M (2021) Orthostatic intolerance in post-concussion patients. Phys Sportsmed 1-6. https://doi.org/10.1080/00913847.2021.1953357

Jacob G, Costa F, Shannon JR, Robertson RM, Wathen M, Stein M, Biaggioni I, Ertl A, Black B, Robertson D (2000) The neuropathic postural tachycardia syndrome. N Engl J Med 343(14):10081014. https://doi.org/10.1056/NEJM200010053431404

Lauria G, Bakkers M, Schmitz C, Lombardi R, Penza P, Devigili G, Smith AG, Hsieh ST, Mellgren SI, Umapathi T, Ziegler D, Faber CG, Merkies IS (2010) Intraepidermal nerve fiber density at the distal leg: a worldwide normative reference study. J Peripher Nerv Syst 15(3):202-207. https://doi.org/10.1111/j.1529-8027. 2010.00271.x

Lo YL, Leong HN, Hsu LY, Tan TT, Kurup A, Fook-Chong S, Tan BH (2005) Autonomic dysfunction in recovered severe acute respiratory syndrome patients. Can J Neurol Sci 32(2):264. https://www. ncbi.nlm.nih.gov/pubmed/16018168
Raj SR, Arnold AC, Barboi A, Claydon VE, Limberg JK, Lucci VM, Numan M, Peltier A, Snapper H, Vernino S, American Autonomic S (2021) Long-COVID postural tachycardia syndrome: an American Autonomic Society statement. Clin Auton Res 31(3):365-368. https://doi.org/10.1007/s10286-021-00798-2

Romero-Sanchez CM, Diaz-Maroto I, Fernandez-Diaz E, SanchezLarsen A, Layos-Romero A, Garcia-Garcia J, Gonzalez E, Redondo-Penas I, Perona-Moratalla AB, Del Valle-Perez JA, Gracia-Gil J, Rojas-Bartolome L, Feria-Vilar I, Monteagudo M, Palao M, Palazon-Garcia E, Alcahut-Rodriguez C, SopelanaGaray D, Moreno Y, Ahmad J, Segura T (2020) Neurologic manifestations in hospitalized patients with COVID-19: the ALBACOVID registry. Neurology 95(8):e1060-e1070. https:// doi.org/10.1212/WNL.0000000000009937

Schondorf R (1993) New investigations of autonomic nervous system function. J Clin Neurophysiol 10(1):28-38. https://doi.org/10. 1097/00004691-199301000-00004

Shouman K, Vanichkachorn G, Cheshire WP, Suarez MD, Shelly S, Lamotte GJ, Sandroni P, Benarroch EE, Berini SE, CutsforthGregory JK, Coon EA, Mauermann ML, Low PA, Singer W (2021) Autonomic dysfunction following COVID-19 infection: an early experience. Clin Auton Res 31(3):385-394. https://doi. org/10.1007/s10286-021-00803-8

Sletten D, Grandinetti A, Weigand S, Gehrking T, Gehrking J, Low $\mathrm{P}$, Singer W (2015) Normative values for sudomotor axon reflex testing using QSWEAT ${ }^{\mathrm{TM}}$

Sletten DM, Suarez GA, Low PA, Mandrekar J, Singer W (2012) COMPASS 31: a refined and abbreviated Composite Autonomic Symptom Score. Mayo Clin Proc 87(12):1196-1201. https://doi. org/10.1016/j.mayocp.2012.10.013

Wang C, Song W, Hu X, Yan S, Zhang X, Wang X, Chen W (2021) Depressive, anxiety, and insomnia symptoms between population in quarantine and general population during the COVID-19 pandemic: a case-controlled study. BMC Psychiatry 21(1):99. https:// doi.org/10.1186/s12888-021-03108-2

Publisher's Note Springer Nature remains neutral with regard to jurisdictional claims in published maps and institutional affiliations. 